\title{
Sur quelques Diptères Nématocères du Var, avec la description d'espèces nouvelles de Culicoides (Cératopogonidés)
}

\author{
par J. CALLOT et M. KREMER
}

Le matériel faisant l'objet de cette étude a été recueilli dans le sud du département du Var durant le mois de juin. Les méthodes de captures et les biotopes seront indiqués à propos des différentes familles étudiées.

\section{PSYCHODIDAE}

\section{Phlebotomus (Sergentomyia) minutus (Rondani).}

Une seule femelle capturée au piège lumineux dans un parc à chênes-lièges, eucalyptus et citronniers, juin 1964 ; le Canadel. La diagnose de cette espèce très répandue sur le bord méditerranéen de l'Europe pose peu de difficultés. Nous avons cependant été quelque peu surpris par l'aspect inhabituel du canal spermioducte qui est très dilaté sur cet exemplaire, presque autant que la spermathèque elle-même.

\section{CULICIDAE}

Tous les Culicidae ont été obtenus à l'état larvaire, puis élevés jusqu'au stade adulte. Les gittes larvaires étaient constitués par des «trous de sorcières » ou « marmites de géants », dépressions cupuliformes creusées par les eaux des ruisseaux dans les roches tendres.

Les gîtes étudiés sont entre la Garde-Freinet et Vidauban, à la jonction des routes N. 558 et D. 48 , sur un affluent de l'Aille. A l'exception d'un, ils sont tous exposés en plein soleil (cf. Callot, 1962).

Les espèces trouvées sont:

1) Aedes (Stegomyia) vittatus (Big.).

Nombreuses marmites de géants; généralement seul, associé une fois à Culex hortensis.

2) Anopheles du groupe maculipennis.

Une marmite de géants, associé à C. pipiens.

Annales de Parasitologie humaine et comparée (Paris), t. 40, 1965, $\mathrm{n}^{\circ} 3$ 
3) Culex (Culex) pipiens pipiens (L.).

Plusieurs gîtes, associé soit à $A$. maculipennis, soit à $C$. impudicus et $C$. hortensis. 4) Culex (Neoculex) hortensis Fic.

Trouvé seul dans une marmite de géants, abritée sous des broussailles; dans une marmite non abritée et associé soit à Aedes vittatus, soit à C. impudicus et $C$. pipiens.

5) Culex (Neoculex) impudicus Fic.

Un gîte, associé à $C$. hortensis et $C$. pipiens.

\section{CERATOPOGONIDAE CULICOIDES}

1. Culicoides derisor n. sp.

Trois mâles et deux femelles capturés au piège lumineux, 15 et 16 juin, Le Canadel (même localité et conditions que pour Phlebotomus minutus).

\section{Mâle.}

Espèce de petite taille.

Thorax brun; abdomen blanchâtre avec des tergites plus sombres. Dessins du thorax non observés sur ces spécimens qui n'ont pu être examinés à sec. Scutellum sensiblement de la même teinte que le scutum. Haltères blancs.

Pattes blanchâtres à très légèrement brunâtres, présence d'un anneau plus clair à la base des tibias.

Peigne tibial postérieur à quatre soies dont les longueurs sont (chez un paratype) : $32,32,26,20 \mu$.

\section{AIle :}

Pas de taches visibles, couleur de fond très légèrement grisâtre, parfaitement transparente. Présence de longues soies noires sur les nervures radiales; macrotriches dans l'ensemble peu nombreuses, totalement absentes de la cellule basale, sous-costale ; aucune (type) à deux macrotriches (paratype) entre nervure $\mathrm{M}$ et cubitale ; aucune (type) à six (paratype) dans la cellule anale.

Longueur de l'aile : $0,63 \mathrm{~mm}$; largeur : $0,27 \mathrm{~mm}$.

Longueur de la costa : $0,28 \mathrm{~mm}$ (à partir de l'arculus).

Lobe postérieur de l'aile très peu marqué, la plus grande largeur de l'aile étant bien au-delà des cellules radiales.

\section{TÊTE :}

Antennes brun-blanchâtre; scape volumineux et brun foncé; quatre derniers articles plus bruns à leur partie distale et à base blanche. Insertion des longues soies entre la moitié et le tiers proximal sur les articles courts. 

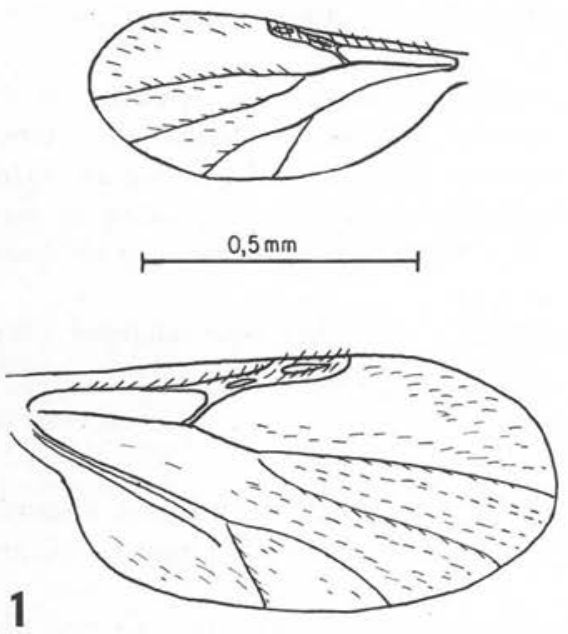
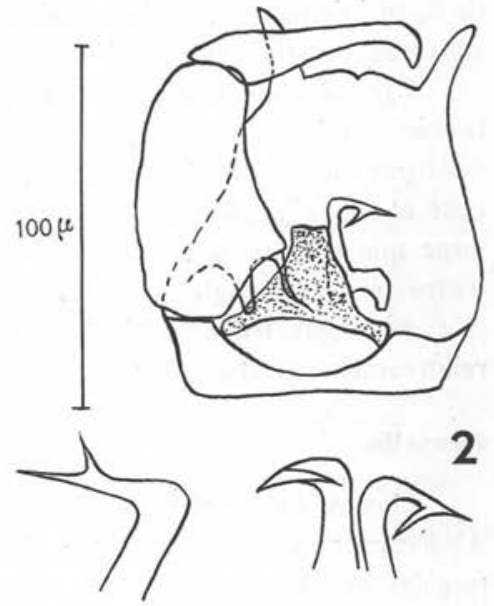

Fig. 1. - Culicoides derisor n. sp. Ailes du mâle et de la femelle

Fig. 2. - Culicoides derisor. Hypopygium du $\sigma^{x}$. (L'aedeagus est schématisé). Extrémité distale des paramères

Fig. 3. - Culicoides derisor. Aedeagus

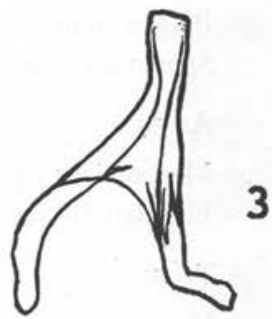

Premier article long, en fût de canon, le troisième en pomme de pin, le second de forme intermédiaire entre les deux précédentes.

Longueur des articles et répartition des sensilles :

$\begin{array}{lllllllllllllll}\text { Articies } & \ldots \ldots \ldots \ldots \ldots & 3 & 4 & 5 & 6 & 7 & 8 & 9 & 10 & 11 & 12 & 13 & 14 & 15\end{array}$ $\begin{array}{lllllllllllllll}\text { Longueur en } \mu & \ldots \ldots & 58 & 24 & 20 & 22 & 22 & 20 & 20 & 20 & 16 & 23 & 72 & 48 & 56\end{array}$

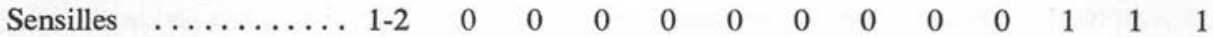

Palpe blanchâtre ; troisième article modérément renflé ; fossette sensorielle petite et ronde.

Longueur des articles (en $\mu$, premier et second comptés ensemble) : 30, 36, 20, 24.

\section{HYPOPYGIUM :}

Tergite à peine échancré, à bord convexe; processus digitiformes divergents et longs.

Sternite échancré en pente douce sur la moitié de sa longueur; membrane basale nue.

Coxites renflés dans leur tiers basal; apodème ventral long, assez fin, en forme 
de $\mathrm{S}$, se joignant sur la ligne médiane ; apodème dorsal digitiforme bien chitinisé. Style sans particularité, extrémité recourbée en bec.

Paramères à base de forme habituelle à double renflement, relativement bien chitinisée, suivie d'une partie rectiligne. Tiers distal recourbé vers l'extérieur et se terminant par une pointe de modérément à très fortement effilée. Partant presque de l'extrémité et se dirigeant vers l'intérieur, existe une pointe dont la base d'insertion est aussi large que le paramère à cet endroit. L'ensemble a un aspect de harpon ou de queue d'aronde selon l'angle de la préparation.

Aedeagus trapézoïdal, légèrement en gouttière ; assez fortement chitinisé ; bras relativement courts.

\section{Femelle.}

Thorax brun, plus sombre que chez le mâle; dessins du thorax non observés. Abdomen prenant une teinte légèrement rougeâtre dans le milieu de Grenier ; plaques tergales brunâtres.

Pattes brunâtres, plus sombres que celles du mâle; base des tibias un peu plus claire ; esquisse de zones plus claires aux extrémités des fémurs.

Peigne tibial à quatre soies dont les longueurs sont : $48,48,40,34 \mu$.

Spermathèques: une rudimentaire et deux ovoïdes subégales de 52 et $48 \mu$.

\section{AILES :}

Macrotriches plus abondantes que chez le mâle et à lobe postérieur plus prononcé. Longueur : 0,79 mm ; costa : 0,48 mm (à partir de l'arculus); largeur : 0,40 mm.

\section{TÊTE :}

Yeux séparés et réunis par une suture simple. Largeur de l'écartement sur la longueur de faces opposées: $1 / 3$.

Présence de dents sur les mandibules et les maxilles.

Palpe. Deux premiers articles blanc-brunâtre, troisième article un peu plus foncé, comme les deux derniers. Troisième article bien renflé, de forme trapézoïdale ; fossette ronde et petite, à bords légèrement surélevés. Longueurs des articles : 44, 45, 22, $22 \mu$ म́ (deux premiers articles comptés ensemble).

Antennes. Scape brun foncé, tous les autres articles bruns. Premier article en massue, les suivants globuleux, puis plus allongés. Articles 11 à 14 cylindriques à col distal rétréci, $15 \mathrm{~cm}$ en forme de pomme de pin. Présence d'une bande légèrement plus claire au niveau des insertions des longues soies.

$\begin{array}{lrrrrrrrrrrrrrr}\text { Articles } & \ldots \ldots \ldots \ldots & 3 & 4 & 5 & 6 & 7 & 8 & 9 & 10 & 11 & 12 & 13 & 14 & 15 \\ \text { Longueur } & \ldots \ldots \ldots \ldots & 40 & 24 & 25 & 28 & 28 & 28 & 28 & 30 & 52 & 52 & 56 & 56 & 68 \\ \text { Nombre de sensilles . . } & 2,5 & 0 & 0 & 0 & 0 & 0 & 0 & 0 & 1 & 1 & 1 & 1 & 2\end{array}$

Longueur totale : $515 \mu$; indice : 1,23 .

Cette espèce est facile à distinguer des espèces décrites récemment grâce à la forme des paramères en harpon. $C$. albihalter s'en distingue par l'étroitesse de ses cel- 
lules radiales. $C$. latipennis est de taille beaucoup plus grande. Les yeux chez $C$. nanulus sont séparés par une bande très étroite.

Par son aspect général et par la forme de son hypopygium, également par la disposition des sensilles des femelles, cette espèce est à placer dans le groupe de $C$. heliophilus.

Type, cotypes et paratypes déposés à l'Institut de Parasitologie de la Faculté de Médecine de Strasbourg.

2. C. obsoletus (Meigen).

Une femelle capturée au piège lumineux ; le Canadel, 10-6-64.

3. C. maritimus Kieffer.

Deux femelles capturées au piège lumineux le 12-6-64, et un mâle et une femelle le 16-6-64; le Canadel.

4. C. begueti Clastrier.

Deux femelles capturées au piège lumineux le 12-6-64; une femelle le 15-6-64 ; trois femelles le 16-6-64; le Canadel.

5. C. sylvarum Callot et Kremer.

Une femelle capturée au piège lumineux le 16-6-64 ; le Canadel.

6. C. halophilus Kieffer.

Une femelle capturée au piège lumineux le 16-6-64; le Canadel.

7. C. heteroclitus Kremer et Callot (1).

Neuf mâles et quatre femelles obtenus à partir de boues conservées au laboratoire. Prélèvement effectué le 30 juin 1964, entre le Canadel et La Mole, au bord de la rivière La Mole, sous un pont de la route D. 27. Eclosions dans le courant des mois de juillet et août.

Espèce de taille moyenne, de couleur brunâtre-bistre. Il faut noter d'emblée que cette espèce est difficile à classer à cause de certaines particularités morphologiques du mâle, tant de son hypopygium que de ses ailes. Nous avons même hésité un moment à en faire un nouveau genre. En raison cependant des femelles, nous le considérons provisoirement comme un bon Culicoides.

Mâle.

Thorax brunâtre à prunosité mordorée; scutellum plus clair que le scutum ; abdomen grisâtre ou vert-de-gris, à bandes plus foncées.

Pattes brunâtres, tous les genoux noirâtres; bandes claires peu visibles à la base des fémurs des deux premières paires de pattes et des tibias correspondants (constant seulement pour la première paire de pattes). Peigne tibial postérieur à quatre soies principales dont les longueurs sont : 44, 48, 40, $36 \mu$.

(1) Le mâle de cette espèce a été décrit, sous la forme que nous reproduisons ici, au $1^{\text {er }}$ Congrès international de Parasitologie, Rome, 1964 (sous-presse). 

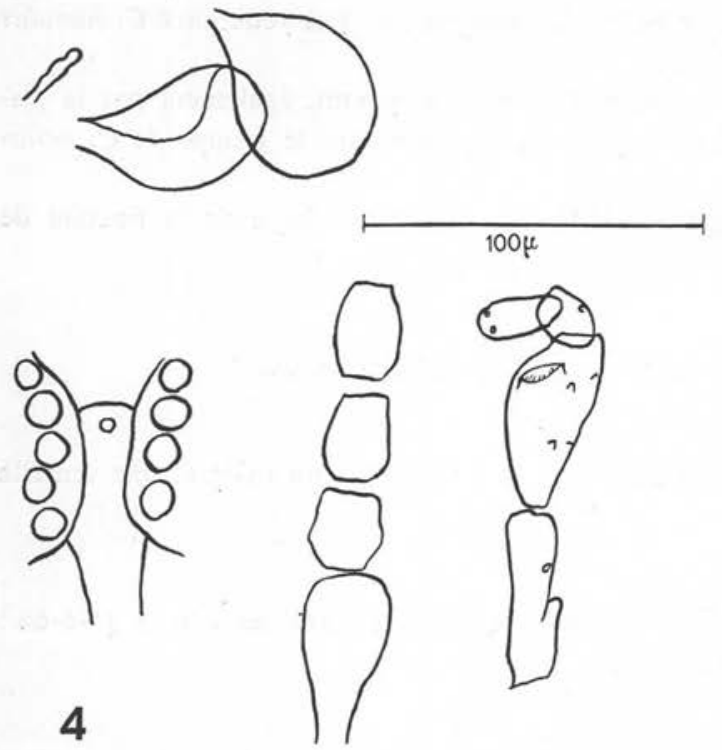

FIG. 4. - Culicoides derisor $\$$ : Spermathèque, espace interoculaire, premiers articles au flagelle; palpe

FIg. 5. - Culicoides heteroclitus : Ailes du mâle

FIc. 6. - Culicoides heteroclitus : Ailes de la femelle

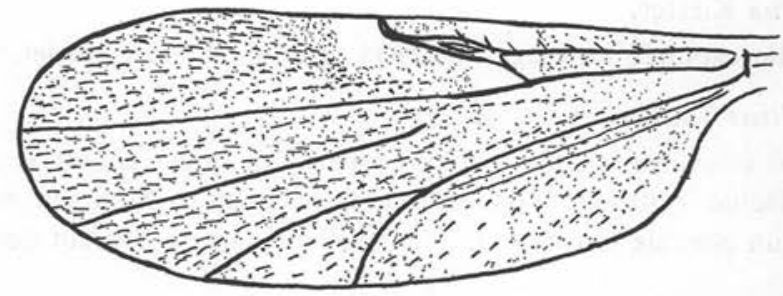

5

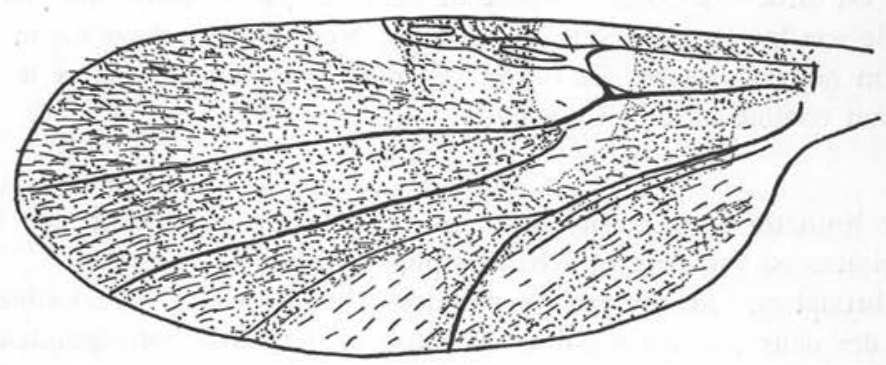

6

AILE :

Couleur de fond très légèrement grisâtre. Taches claires disposées comme suit :

- une táche sur $\mathbf{T}$, première cellule radiale et un peu sur $\mathbf{M}$; 
- une tache triangulaire petite après la seconde cellule radiale (n'entamant pas cette cellule) ;

- une tache à la base de l'aile, peu nette, se prolongeant sous $\mathrm{Cu}$;

- une tache à la partie distale de la cellule anale ;

- une tache dans la cellule cubitale ;

- des ébauches de taches, à peine marquées aux extrémités des cellules $\mathrm{r} 5, \mathrm{~m} 1$ et $m 2$.

La division de la nervure $\mathrm{M}$ se fait sous la première cellule radiale. La deuxième cellule radiale n'est pas formée et se réduit comme chez $C$. schultzei à une seule nervure, soit qu'il s'agisse d'une coalescence de $\mathrm{r}$ 4-5 et de $\mathrm{r} 3$, soit d'une absence de $\mathrm{r} 3$ (?). $\mathrm{Ce}$ caractère ne se retrouve pas chez la femelle. Macrotriches longues et abondantes, s'étendant sur toute la surface de l'aile, mais respectant la cellule basale, sous-costale et la partie toute proximale de l'aile. Microtriches comme chez tous les Culicoides.

Longueur de l'aile : 1,05 mm; costa : 0,52 mm (à partir de l'arculus); largeur : $0,5 \mathrm{~mm}$.

\section{TÊTE :}

Scape des antennes volumineux, brun foncé ; article du fouet brun-grisâtre. Premier article claviforme, les suivants arrondis avec un col de plus en plus net distalement. Premier article long à base renflée, puis cylindrique; suivant semblable, mais plus épais, le dernier en forme de pomme de pin.

Antennes :

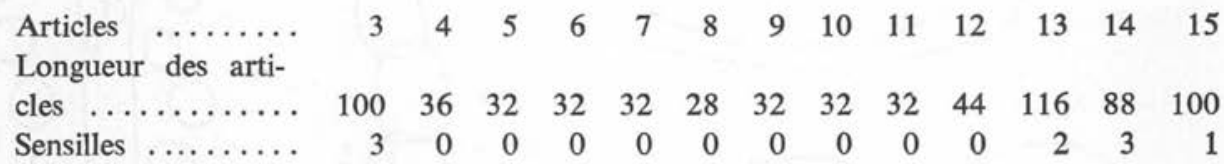

Les sensilles des articles 4 à 10 sont mal observés en raison de l'importance du panache qui est très long et de couleur brune.

Palpe brunâtre ; troisième article bien renflé à fossette sensorielle ronde ; longueur des articles : $56,64,32,32 \mu$.

\section{HYPOPYGIUM :}

Lamelle longue et étroite; bords postérieurs à deux pointes importantes séparées par une fente en $\mathrm{V}$, profonde ; processus longs et cylindriques à extrémité arrondie, mousse et à petit poil du côté interne.

Sternite réduit à une bague mince et fortement chitinisée.

Membrane basale nue.

Coxite très allongé, presque cylindrique; apodème ventral inexistant. Apodème dorsal très chitinisé, plus ou moins digitiforme. Style à base renflée, se continuant distalement, par une touffe de longues soies fortes. Ce style présente deux expansions : la première située entre la moitié et le tiers distal, du côté interne, de forme cylindrique, grosse, longue, arrondie à son extrémité ; la seconde, petite, triangulaire, à la base et du côté interne du style. 

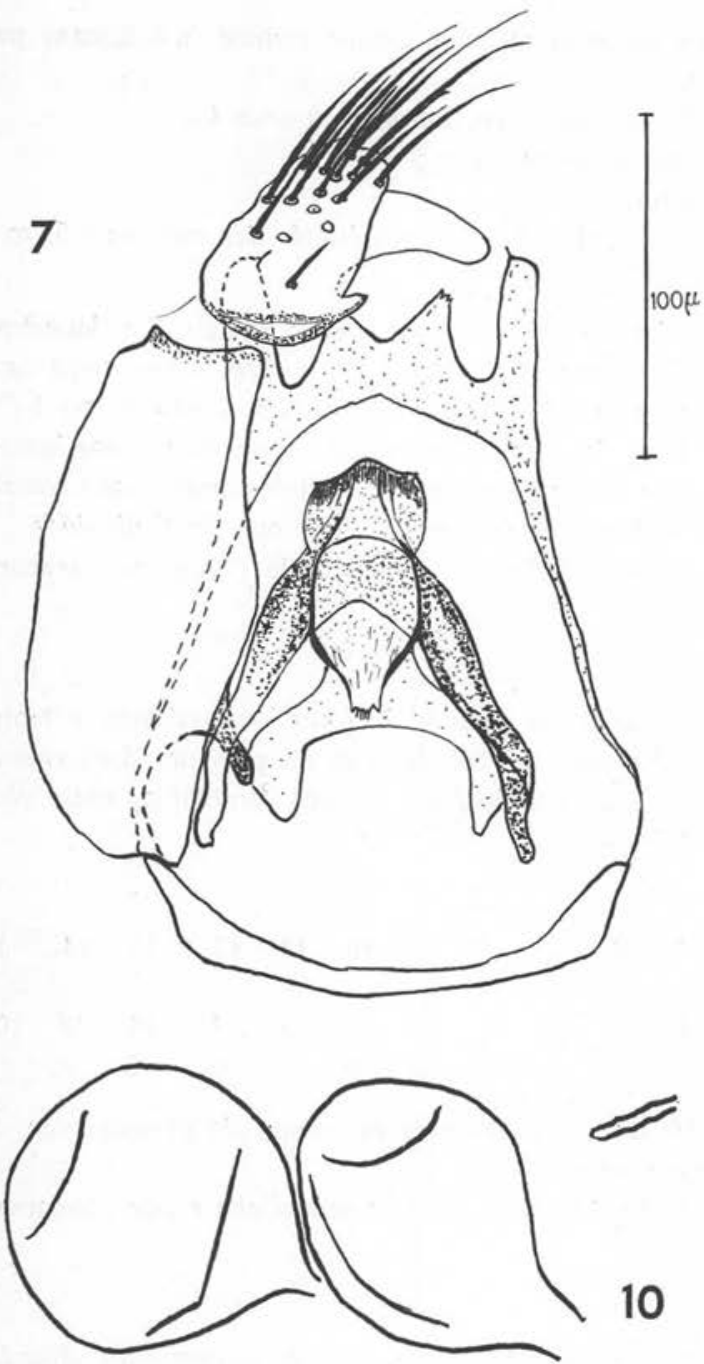

FIG. 7. - Culicoides heteroclitus: hypopygium mâle FIG. 8. - Culicoides heteroclitus : paramères et style FIG. 9. - Culicoides heteroclitus: palpe, premiers articles antennaires et espace interoculaire de la femelle. (L'espace interoculaire de l'exemplaire dessiné ici présente une petite soie surnuméraire d'un côté, qui ne se retrouve pas sur les autres exemplaires)

FIG. 10. - Culicoides heteroclitus: spermathèque
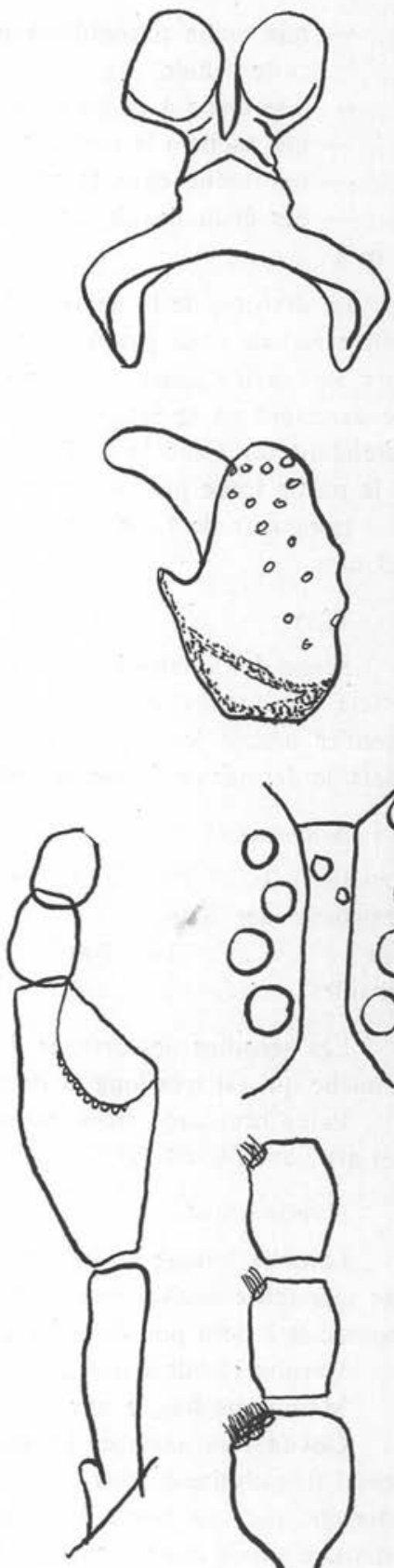

8

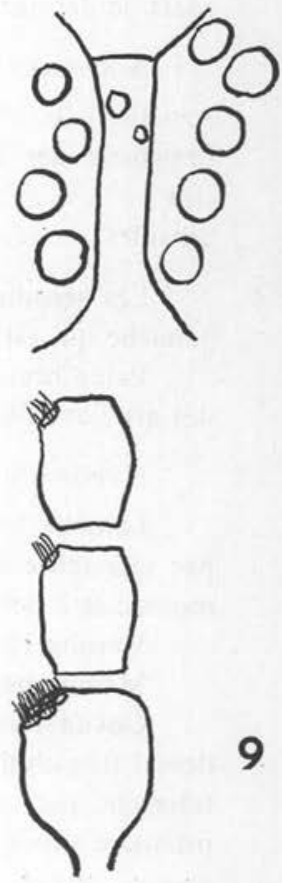


Aedeagus très chitinisé; deux bras aboutissent à un corps en forme d'écusson, strié et à bord distal en forme de double parenthèse bien marquée. En avant de ce « corps», une bande encore bien chitinisée remplit l'angle formé par les bras et le corps ; cette bande elle-même prolongée en avant par un lambeau à contours arrondis, irrégulier se terminant par une petite touffe frangée.

Origine des paramères de forme habituelle, puis soudure parfaite de leur partie médiane en un bloc trapézoïdal ; partie distale de chaque paramère constituée par une sorte de cuillère à manche épais et plicaturé ; partie concave des cuillères située derrière l'aedeagus.

Femelle (décrite ici pour la première fois).

Aspect général identique à celui du mâle.

Pattes de couleur identique ; longueur des épines du peigne tibial : 46, 48, 33, $32 \mu$.

AILES :

Même aspect que celles du mâle; macrotriches plus abondantes encore, mais respectant les mêmes cellules. Ici la deuxième cellule radiale est bien formée ; légèrement plus petite que la première. La nervure $\mathrm{r} 3$ est bien présente, quoique moins en relief que les autres nervures; disposition des taches alaires semblable à celle du mâle. Longueur de l'aile : $1,15 \mathrm{~mm}$; costa : 0,62 mm (compté à partir de l'arculus); largeur : $0,64 \mathrm{~mm}$.

\section{TÊTB :}

Yeux nus, séparés par des bords parallèles, une suture au-dessus du poil médian, simple, droite (l'exemplaire dessiné présente un poil surnuméraire entre les deux yeux).

Des dents sur les maxilles et les mandibules.

Antennes: articles d'un brun clair, à base blanche. Articles courts en forme de tonneaux, sans col bien marqué ; articles 11 à 14 en fût de canon, à col indiqué, le quinzième plus ou moins cylindrique, puis distalement tronconique.

\begin{tabular}{|c|c|c|c|c|c|c|c|c|c|c|c|c|c|c|}
\hline & & 3 & 4 & 5 & 6 & 7 & 8 & 9 & 10 & 11 & 12 & 13 & 14 & 15 \\
\hline a & $\ldots \ldots$ & 7 & $1^{*}$ & $1 *$ & 1 & & 0, & 1 & 1 & & & & & \\
\hline on & & 45 & 28 & 30 & 32 & 32 & 31 & 32 & 32 & 50 & 52 & 60 & 65 & 93 \\
\hline
\end{tabular}

Longueur totale : $582 \mu$; indice antennaire : 1,22.

Palpe brun, très semblable à celui du mâle ; troisième article renflé dans son tiers distal, fossette ovalaire, occupant le tiers distal, à bords légèrement surélevés. Partie toute distale des quatre premiers articles blanche, dernier article entièrement brun.

Longueur des articles : 76, 84, 24, $28 \mu$ (deux premiers articles comptés ensemble).

Spermathèques globuleuses à col étroit, l'une plus large que l'autre; mesurant l'une 80 sur $52 \mu$ et l'autre 76 sur $76 \mu$; une spermathèque rudimentaire en forme de doigt de gant.

\footnotetext{
* Parfois deux sensilles sur ces articles.
} 
L'aspect tout à fait étrange du mâle de cette espèce ne permet aucune hésitation en ce qui concerne sa diagnose. La femelle, d'aspect fort banal, peut être éventuellement difficile à distinguer de $C$. jurensis Callot, Kremer et Déduit, qui présente plus de sensilles. $C$. begueti Clastrier a un palpe différent ; $C$. cubitalis a un indice antennaire plus élevé.

Il est difficile de classer cette espèce dans un groupe de Culicoides; par ses paramères elle est à rapprocher du groupe nubeculosus dont la sépare cependant, la présence de deux spermathèques chez la femelle. Il paraît préférable actuellement de ne pas classer cette espèce.

Type, cotype et paratype déposés à l'Institut de Parasitologie de la Faculté de Médecine de Strasbourg.

\section{C. cubitalis Edwards.}

Un mâle et une femelle sont éclos d'un prélèvement de boues effectué sur un petit affluent de La Mole, très près du lieu d'où est éclos $C$. heteroclitus.

\section{C. longipennis Khalaf.}

Un mâle et une femelle éclos de boues prélevées le long de La Mole, près du village de La Mole, deux femelles écloses du même gîte que les $C$. cubitalis, une femelle et un mâle éclos du gîte à $C$. heteroclitus.

La diagnose de cette espèce est assez facile (sauf avec $C$. similis), l'hypopygium est typique : aedeagus à extrémité effilée, terminée par une petite touffe de poils, paramères du type de $C$. accraensis. Les femelles ont des sensilles sur les huit premiers articles du fouet antennaire et pas de sensille sur les cinq derniers.

A notre connaissance, cette espèce n'a été signalée jusqu’à présent qu'en Irak et en Azerbaïdjan.

Nous avions signalé avec Coluzzi, la présence en Italie de $C$. similis; les exemplaires que nous avons recueillis dans le Var et que nous considérons tout au moins provisoirement comme les $C$. longipennis, sont en fait très proches de $C$. similis. L'extrémité de l'aedeagus des deux lots présente des variations, que nous ne pouvons systématiser sur si peu d'exemplaires. Il en est de même pour les apodèmes ventraux, ceux d'Italie étant plus proches de ceux de $C$. similis, ceux du Var de $C$. longipennis. Il faudra attendre d'avoir de nouveaux matériaux pour trancher le problème longipennissimilis.

10. C. sp. Un mâle et cinq femelles, appartenant au groupe odibilis, ne présentent pas suffisamment d'originalité pour être décrits comme nouveaux. Nous ne pouvons cependant, pour l'instant, les rapporter à aucune espèce connue.

\section{Résumé}

Nous rapportons l'existence, dans le Massif des Maures (Var), des espèces suivantes de Nématocères: Phlebotomus minutus, Aedes vittatus, Anopheles maculipennis, Culex pipiens pipiens, Culex hortensis et Culex impudicus. Les Culicidae ont été trouvés à 
l'état larvaire dans des marmites de géants, le phlébotome a été capturé au piège lumineux.

Par piège lumineux et par culture de boues prélevées dans la région littorale des Maures, nous avons trouvé les espèces suivantes de Culicoïdes : $C$. obsoletus, $C$. maritimus, C. begueti, C. sylvarum, C. halophilus, C. cubitalis, C. longipennis, C. heteroclitus, C. derisor n. sp.

C. longipennis est signalé pour la première fois et ses rapports avec $C$. similis sont discutés sans être définitivement résolus. Une espèce est laissée en attente.

\section{Bibliographie}

\section{SUR LES Psychodidae.}

Theodor (O.), 1958. - Psychodidae-Phlebotominae in Lindner \& Die Fliegen des Palaearktischen Region; Fascicule 201. Stuttgart.

\section{SUR LES Culicidae.}

Callot (J.), 1962. - Nouvelle localisation française d'Aedes vittatus Bigot. Ann. Par. Hum. Comp., 37, 174.

- et DAo VAN Ty, 1942-1943. - Localités françaises nouvelles pour Culex (Culex) theileri, Culex (Barraudius) modestus, Culex (Neoculex) impudicus. Ann. Par. Hum. Comp., 19, 142-150.

Rroux (J. A.), 1958. - Les Culicidés du « Midi » méditerranéen. Encyclopédie entomologique, Paris ; P. Lechevalier.

\section{SUR LES Culicoides.}

CAllot (J.) et Kremer (M.), 1961. - Scission de l'espèce Culicoides truncorum Edwards (Diptera : Ceratopogonidae) en deux espèces. Bull. Soc. Path. Exot., 54, 389-398.

-, KRemer (M.) et Rioux (J. A.), 1963. - Sur des Culicoides (Diptera : Ceratopogonidae) dont une espèce et une variété nouvelles du midi de la France. Ann. Par. Hum. Comp., 36, 121-129.

Clastrier (J.), 1959. - Notes sur les Ceratopogonidés VI. Ceratopogonidés d'Afrique Occidentale française. Arch. Inst. Past. Algérie, 37, 167-197.

Edwards (F. W.), 1939. - Ceratopogonidae in: Edwards, Smart et Oldroyd \& British bloodsucking flies », British Museum, Londres.

GutZevich (A. V.), 1960. - Krovosuscie mokrecy (Diptera: Heleidae). Fauny S.S.S.R. Akad. Nauk S.S.S.R., 18, 72, 130 p.

Dzhafarov (S. M.), 1964. - Krovososuscie mokrecy (Diptera Heleidae) Zakavkazjja Izdateljstvo Akademii Nauk Azerjajdzanskoj S.S.R., 411 p., Baku.

KhalaF (T. K.), 1957. - Heleids from Iraq, with description of new species. Bull. Soc. Ent. Egypte, 41, 335-350.

[Institut de Parasitologie de la Faculté de Médecine de Strasbourg

(Directeur : Professeur J. CALlot)] 